\section{Economy in space}

SIR-Your leading article "Economy in space" (Nature 328, 562; 1987) calls for comment. No doubt what is and is not worthwhile in space deserves scientific evaluation, especially as both this and high-energy physics seem to be coming to the end of their tether. But this is not what the column offers. Instead we have an amazing collection of non sequiturs.

First, if Blue Streak was abandoned because it "would make Britain more vulnerable", why do we now have US missiles on our soil over which we have no control? Further, if Britain has "neglected its technical culture over four decades", how is the "present government attempting to redress the balance", by dismantling higher education?

The fact is that Britain has lost its preeminence in technology through colossal mismanagement and waste of human resources. Some of us who worked on the scientific war effort and moved to industry have seen this at first hand, finding it impossible to do anything. Later, having emigrated to the other side of the Atlantic, one saw this even more clearly.

Bashing Labour, and now academics, is not going to solve the problem, nor is monetarism, which gives only the appearance (fragile indeed) but not the substance of prosperity. In fact the fear of inflation, referred to in the column, was due precisely to the chancellor having allotted the budget surplus to tax rebate rather than to education and research. Apart from their shortsightedness, these policies have simply given a new twist to the polarization (us and them) that has been the main source of our problems in the first place. True we do have a cultural crisis, but it is not what the column indicates.

M. C. Goodall

3 Robson Terrace, Shincliffe Village,

Durham DH1 2NL, UK

\section{Databases}

SiR-C.O. Pabo's letter ${ }^{1}$ on molecular biology databases is pertinent; his proposals have been considered at one time or another by those involved in maintaining sequence databases. His "higher order databases" (consisting of structural motifs, similarities to other sequences and the like) are feasible now.

Yet there is no consensus among molecular biologists and the agencies that fund such work that such an advanced database is needed; nor is there support for such databases among the organizations responsible for funding the national sequence databases. My understanding is that the financial support for GenBank ${ }^{2}$ involves the National Institute of General Medical Sciences; the National Library of Medicine; the National Cancer Institute; the National Institute of Allergy and
SIR-Your leading article "Economy in space" (Nature 328, 562; 1987) shows a surprising superficiality.

It is your prescription that gives cause for concern. You are right to identify the dilemma that exists for this or any advanced nation regarding its role in space or in any scientific or development activity. But Britain has an advanced economy together with a well-established expertise in space and while much of your previously expressed opinion has concentrated on how to maintain this situation, you are now counselling a national descent to underdeveloped status.

The demise of Blue Streak as a purely British project was indeed inevitable. The inability of Britain to secure a worthwhile involvement in the later successful adoption of that technology in the Ariane programme was not. Britain and other European countries, including Belgium, can through the European Space Agency (ESA) continue to share in a worthwhile European role in space at a cost that each can afford. Britain's relatively minor part in the highly successful Ariane programme followed from the confused and fragmented space policy of successive governments. The formation of the British National Space Centre and the arrival of a space professional such as Roy Gibson to head it were signs that at last a UK government was determined on an effective and coordinated space policy. As the main ESA programme is an optional one, the establishment and operation of such a policy can provide an excellent defence against involvement in extravagant or unrealistic projects.

A range of communication satellites for civil and defence use, a variety of microwave and other sensors together with a demonstrated and world leading position in ground control systems, navigation systems and software are distinctive products of high added value that form an important part of an industrial sector with a turnover of more than $£ 2,000$ million. The United Kingdom's leading role in constructing the Giotto spacecraft which passed within $500 \mathrm{~km}$ of the nucleus of Halley's comet surely demonstrates a substantial level of technical competence. Roy Gibson's message to the government was that the industrial returns from our present modest ESA programme could be most effectively enhanced by increasing the complementary UK activity by the equivalent of an additional $£ 2$ per UK citizen each year.

This request is far from a demand for a daring role in an ambitious space programme but stems, for example, from a recognition that an involvement in space, in addition to stimulating interest in challenging technological projects, may ultimately be essential to our survival as a species, given our need to monitor, understand, protect and control the climate and the renewable resources of the planet. Increased support of space by industry would be very welcome but is unlikely to be forthcoming for activities which involve a national rather than a corporate return on a timescale of ten or more years.

UK technical people may indeed be undervalued. To "put space on the back burner for a few decades" will do little to increase the perception of the value and importance of technology among ministers, the general public or even the engineers and scientists themselves. Once specialist skills and expertise are lost, particularly if established teams are broken up, they are impossible to resurrect. If we wait two or three decades it will be much too late to repeat Gibson's question.

J. LeOnARD CUlhane

Mullard Space Science Laboratory,

University College London,

Holmbury St Mary,

Dorking,

Surrey RH5 6NT, UK
Infectious Diseases; the National Institute of Arthritis, Diabetes, and Digestive and Kidney Diseases; the Division of Research Resources at the National Institutes of Health; the National Science Foundation; the Department of Energy; the Department of Agriculture; and the Department of Defense. With 10 organizations supporting the effort, we are still left with a database whose utility is limited because essential information (strain or species-specific variation in sequence, for example) is not accessible or, frequently, not even included.

The problem does not lie with the organizations doing the data collecting; they are understaffed as a result of the meagre commitment of the foregoing organizations relative to the size of the problem. What is needed is (1) a commitment of increase resources (chiefly in staffing) to the databanks; (2) a require- ment by granting agencies that sequence data be submitted in machine-readable form to the databanks as a condition of funding; and (3) standardization of database design among the national databases.

In practice, each entry added to the genetic sequence databanks represents a loss of information; who would go through the 14,000-or-so literature citations and add the annotation to the sequences already present in the dababanks? To prevent such loss of this information, the annotation and submission must become the researcher's responsibility.

Department of Biochemical Daniel Davison and Biophysical Sciences,

University of Houston,

Houston, Texas 77004, USA

1. Pato. C.O. Nature 327, 467: 1987

. GenBank Floppy Diskette User's Guide, Release 48.0. February 1987 . 